\title{
MATERNIDADE PRECOCE: UMA DAS CONSEQÜENCIAS DO ABANDONO ESCOLAR E DO DESEMPREGO
}

\section{PREMATURE PREGNANCY: ONE OF THE CONSEQUENCES OF SCHOOL DROPOUT AND UNEMPLOYEMENT}

Ana Lucia Barreto da Fonseca *
Neuraci Gonçalves de Araújo **

Fonseca ALB, Araújo NG. Maternidade precoce: uma das conseqüências do abandono escolar e do desemprego. Rev Bras Cresc Deserzv Hum 2004;14(2): 21-25.

\begin{abstract}
Resumo: Este estudo teve como objetivos conhecer a condição de educação e trabalho das jovens que vivenciam a maternidade precoce e estabelecer uma relação entre gravidez adolescente e abandono escolar, comparando a realidade educacional e de trabalho de jovens mães de comunidades da Bahia e Sergipe. Para tanto, foram realizadas visitas domiciliares à oitenta adolescentes gestantes ou mães em comunidades carentes das duas capitais. Os resultados apontaram que mais da metade já estavam fora da escola quando engravidaram, e muitas deixaram a escola após saber da gestação. Poucas continuavam os estudos, mesmo tendo uma rede de suporte favorável. A maioria das adolescentes não trabalhava antes de engravidar, algumas passaram a trabalhar após a maternidade, a maioria exercendo atividades domésticas. Pode-se supor que a educação não esta vinculada à cultura dessas comunidades. O valor social da mulher se restringe ao exercício da maternidade, valorizado no grupo, e que Ihe institui 0 status de adulto. A maternidade precoce, portanto, denota ser mais uma consequência do abandono social a que as jovens de classe baixa estão sujeitas do que provocadora de uma exclusão.
\end{abstract}

Palavras-chave: Maternidade. Adolescência. Escolarização. Trabalho.

\section{INTRODUÇÃO}

A adolescência, mesmo sendo um tema tratado nos meios acadêmicos desde o início do século XX, nunca suscitou tantas pesquisas como nas últimas décadas, sendo objeto de estudo das ciências da saúde e da educação, na busca de compreender as várias nuances dessa fase do desenvolvimento.

Hoje, pensa-se e fala-se sobre adolescência em todos os segmentos da sociedade. As mudanças que ocorreram nas estruturas familiares e na sociedade como um todo tornaram mais visíveis algumas das difíceis questões que abarcam tal tema. A adolescência é, em geral, relacionada a conflitos e dificuldades, seja na família, na escola e nas demais instâncias da sociedade, e por isto tornou-se objeto de estudos que buscam conhecer e explicar os seus determinantes ${ }^{1}$.

Fávero e Mello $^{2}$ definem que muitas das alterações ocorridas no contexto sócioeconômico no século XX, e as conseqüentes mudanças nas concepções de família, de criança, de juventude, de educação de filhos, interferiram diretamente no comportamento dos indivíduos e, mais efetivamente, no dos jovens. Os pais já não têm tanta clareza das normas e valores que norteiarn as relações familiares e o ambiente sócioeconômico cada vez exige mais adequação às necessidades mercadológicas.

Essa instabilidade promove o aumento da insegurança, da dúvida, do questionamento, da quebra dos valores, já tão presentes na adolescência, ao mesmo tempo que distancia a autono-

\footnotetext{
Mestra em Educação (UFBA), Psicóloga, professora da Universidade Tiradentes, Aracaju, Sergipe. Av. Augusto Franco, 2000. Qd. I0/Lt.05 Siqueira Campos. Aracaju/SE. anaelara@ig.com.br

** Psicóloga. neuracipsi@bol.com.br
} 
mia e a independência tão almejadas pelos jovens. Essa recente dinâmica da sociedade tem sido responsabilizada por muitas das dificuldades em que se envolvem os adolescentes, como a marginalidade, a violência, a liberdade sexual, o uso de drogas, e a gravidez na adolescência.

A gravidez na adolescência, entre muitas das dificuldades enfrentadas pelos jovens e por suas famílias, provoca também o receio, por parte da sociedade e das autoridades de saúde e de educação, quanto às conseqüências deste fenômeno para as jovens e para os seus filhos. Tal problemática é, em geral, relacionada a comprometimentos na vida das jovens mães e de seus bebês, principalmente no que se refere ao abandono escolar e a conseqûente dificuldade de inserção no mercado de trabalho ${ }^{2}$.

Sob este prisma, a maternidade na adolescência é vista como um fator que altera o ciclo natural do desenvolvimento das jovens, justificado pelo falo delas terem que assumir um novo papel, o de mãe, conjugado, algumas vezes, com o de esposa, de modo brusco e definitivo, muitas vezes incompatível com a condição de adolescente. Há o pressuposto de que a gravidez precoce provoca uma ruptura no ciclo desenvolvimental das adolescentes, comprometendo seu crescimento em várias instâncias, principalmente nos estudos, com implicações no futuro profissional.

As expectativas contemporâneas em relação às adolescentes são de que a maternidade só deve ocorrer após a mulher concluir alguns estágios da vida adulta: os estudos, a profissão, o emprego, o casamento, a casa. Antes disso, esse evento provoca uma ruptura no processo de desenvolvimento da família como um todo, pela expectativa de que, com a evasão escolar, a filha mantenha-se dependente da família ${ }^{3}$.

A gravidez adolescente passa a ser vista como a grande responsável pelo abandono escolar das jovens, o que gera, ao longo do desenvolvimento das mulheres, uma condição de subsistência precária, mantendo-as em situações sócio-econômicas desfavoráveis.

No entanto, para Esteves ${ }^{4}$ e Catharino e Griffin $^{5}$, a gravidez precoce não deve concentrar esta discussão: as jovens das classes populares tornar-se-iam mães precocemente exatamente pela baixa expectativa quanto à escolaridade e a possibilidade de exercerem atividades melhor remuneradas, definindo a maternidade como o principal papel a ser representado na comunidade, fornecendo o status de mulher adulta.

Esses estudiosos afirmam que as preocupações com a maternidade adolescente geram programas de prevenção e apoio às jovens e seus bebês mas não percebem o quanto este fenômeno é significativo para as garotas, não lhes ofererecendo nenhuma outra garantia, seja de educação de qualidade, seja de trabalho formal. Com isto, constata-se que estes programas não têm atingido seus objetivos, sendo crescente o número de casos de gravidez na adolescência.

A sociedade, ao montar estratégias de prevenção e apoio para informar às jovens, já está tentando conter os riscos que têm sido previstos neste tipo de maternidade, para a mãe e principalmente para o bebê. No entanto, enquanto estas intervenções não estabelecerem uma relação ecológica, como definida por Bronfenbrenner ${ }^{6}$, de modo a explicitar que há inter-influências entre as estruturas sociais, estarão fadados ao fracasso. De fato, para compreender os determinantes do comportamento humano, é necessário conhecer o contexto sócio-cultural, onde o desenvolvimento resulta de um processo inter-relacional no qual os micro sistemas indivíduo, família, comunidade determinam e são determinados pelos macro sistemasideologias, valores e normas culturais,

Em estudo com mães adolescentes de classes sociais desfavorecidas, Fonsêca ${ }^{7}$ observou que a gravidez precoce era encarada com naturalidade pela comunidade. Esperava-se que a garota engravidasse assim que começava a se relacionar com o sexo oposto; caso isto não ocorria, a jovem passava a se sentir diferente das demais e, em alguns casos, buscava estratégias para alcançar este objetivo. Em geral, elas tinham conhecimento dos comprometimentos sócio-desenvolvimentais a que estavam sujeitas, mas atribuíam o valor social das mulheres ao exercício da maternagem e às dificuldades como inerentes a isso.

Em um contexto cultural que coloca a maternidade como principal papel social exercido pela mulher, ao reiterar a prática da maternidade precoce, a jovem apenas reproduz os valores e normas culturais do seu grupo social, assim como de seus antecedentes, manifestando, deste modo, a sua representação da maternidade ${ }^{5}$.

Nas mães adolescentes entrevistadas por Fonsêca ${ }^{7}$ o nível de escolaridade foi baixo; porém, o atraso escolar nessas populações pode ser visto como um problema crônico. Ficou evidenciado, igualmente, que a gravidez dificulta a retomada da escolaridade, principalmente devido à pouca expectativa em relação à mesma como um fator de mudança e melhoria de vida.

A gravidez concorre, deste modo, para agravar a realidade, já bastante precária, dessas jovens, que se vêem excluídas de qualquer possibilidade de ascensão social por meio da escolarização, tendo a expectativa de que a união com um parceiro e a maternidade como um recurso para se tornar mulher e melhorar de vida. 
Como afirma Novelino ${ }^{8}$, a maternidade é ainda considerada a principal tarefa da mulher e somente por meio desse papel ela define o seu papel na sociedade. "E a maternidade, hoje opcional, constitui ainda o componente central, definidor da identidade feminina”. Dessa forma, ao se tornar mãe, a garota passa a ser desobrigada de exercer outros papéis sociais, como o de estudante.

Arcieri $^{9}$ argumenta que existe uma distinção entre ser mãe adolescente e ser mãe jovem: segundo o ambiente sócio-cultural onde a jovem esteja inserida, as expectativas relacionadas à escolaridade, profissionalização, e à gravidez estarão definindo os seus comprometimentos e a naturalização do processo. Dessa forma, a gravidez, apesar de não ser desejada ou planejada, é esperada e pode ser o fator que determinará a saída da adolescente da famlia para estruturar a sua casa, cumprindo um ritual de passagem para a etapa adulta - às vezes o único possível em um contexto de exclusão social.

Fávero e Mello² constataram que uma das primeiras coisas que muda na vida das garotas que engravidam é a saída da escola, seja por vergonha ou por terem que assumir um novo papel, o de mulher e mãe. Assim, elas não são estimuladas a estudar e, muitas vezes, não se sentem atraídas para essa função, principalmente nas camadas sociais de baixa renda, onde a expectativa de inclusão social através do estudo é, pelo menos, ambígua.

A maternidade adolescente, alvo de criticas e de programas governamentais com o objetivo de controlá-la, definida como prejudicial às jovens, pode ser a maneira de inserção social encontrada por jovens para quem o sistema educacional não é dirigido nem garante trabalho.

\section{MÉTODOS}

\section{Sujeitos}

Participaram desta pesquisa 80 adolescentes mães e/ou em estado gestacional, 40 de duas comunidades carentes da capital sergipana (Se) e 40 de duas comunidades carentes da capital baiana (Ba), sendo estas últimas divididas em dois grupos, uma pertencente a uma área urbana e outra de uma área semi-rural. Nos dois estados, a maioria das entrevistadas era primípera.

Quanto à faixa etária, observa-se na Tabela 1 a incidência da gravidez é mais precoce em Se, onde 56\% foi mãe antes dos 18 anos de idade para $40 \%$ na Ba. Das adolescentes entrevistadas de Se, 25\% engravidaram entre 13 e 15 anos enquanto que percentual da Ba foi de $10 \%$, com
Tabela 1 - Faixa etária das Adolescentes entrevistadas

\begin{tabular}{ccc}
\hline Faixa Etária & $\begin{array}{c}\text { Bahia } \\
\mathbf{\%}\end{array}$ & $\begin{array}{c}\text { Sergipe } \\
\mathbf{\%}\end{array}$ \\
\hline $13-15$ anos & 10 & 25 \\
16-17 anos & 30 & 31 \\
1 8-19 anos & 32 & 40 \\
20-21 anos & 28 & 05 \\
\hline
\end{tabular}

maior predominância na zona semi-rural. A maioria mora com a família de origem, 77\% em Se e $62 \%$ na $\mathrm{Ba}$, sendo que, em Se, $53 \%$ alteraram a constituição da família de origem trazendo o companheiro para morar com eles enquanto na Ba este falo ocorreu em apenas 13\% (Tabela 2). 44\% haviam constituído seu próprio núcleo familiar com o companheiro, aBa apresentando uma parcela maior de jovens formando um novo agrupamento familiar, principalmente na área semi-rural.

Tabela 2 - Com quem reside a adolescente entrevistada

\begin{tabular}{|c|c|c|}
\hline \multirow{2}{*}{ Com quem mora } & \multicolumn{2}{|c|}{ Bahia Sergipe } \\
\hline & $\%$ & $\%$ \\
\hline Família de origem & 10 & 25 \\
\hline Família de origem e companheiro & 30 & 31 \\
\hline Família nuclear & 32 & 40 \\
\hline Amigos/parentes & 28 & 04 \\
\hline
\end{tabular}

\section{Instrumentos e Procedimentos}

Para coleta de dados foi utilizado um roteiro de entrevista previamente elaborado, de maneira a possibilitar direções variadas conforme a fala das entrevistadas. A coleta dos dados foi realizada na residência das adolescentes, todas localizadas em áreas com populações predominante de baixa renda. $\mathrm{O}$ acesso às adolescentes foi por intermédio da equipe do Projeto "O Programa de Saúde da Família: Condições, Sujeitos e Contextos” em Salvador/Ba e dos Postos de Saúde da comunidade Bugio e da comunidade Soledade em Aracaju/Se. Os dados coletados nas entrevistas semi-estruturadas foram categorizados a partir de respostas similares, facilitando sua análise e interpretação. Terminada essa etapa, foi feita a comparação dos dados coletados entre os dois Estados, Bahia e Sergipe.

\section{RESULTADOS E DISCUSSÃO}

Com o objetivo de verificar a relação entre a maternidade precoce, abandono escolar e desemprego, buscou-se verificar a situação escolar e profissional das entrevistadas quando engravidaram, e como se encontravam no momento. Foi 
levado em consideração, também, quais as expectativas dos sujeitos em relação aos estudos e ao trabalho, e qual a rede de suporte que esta dispunha quanto ao cuidado da criança.

Tabela 3 - Situação Escolar no momento da pesquisa

\begin{tabular}{ccc}
\hline Estuda & $\begin{array}{c}\text { Bahia } \\
\mathbf{\%}\end{array}$ & $\begin{array}{c}\text { Sergipe } \\
\mathbf{\%}\end{array}$ \\
\hline Sim & 27.5 & 20 \\
Não & 72.5 & 80 \\
\hline
\end{tabular}

A Tabela 3 mostra que a maioria das adolescentes não estudava quando foram entrevistadas, $80 \%$ no estado de Se e $72,5 \%$ na Ba. De acordo com o relato das entrevistadas de Aracaju, 92\% delas pararam de estudar quando cursavam o ensino fundamental. e apenas 33\% justificaram a interrupção por causa da gravidez. As outras já haviam abandonado os estudos antes de ficarem grávidas, apresentando os mais variados motivos. Na capital baiana, 90\% das adolescentes que não estudavam, pararam no ensino fundamental, interrompendo os estudos entre a $2^{\mathrm{a}}$ e a $5^{\mathrm{a}}$ série. Dessas, apenas 35\% citaram a gravidez como motivo da interrupção dos estudos e outras (10\%) mencionaram o nascimento do bebê como justificativa. As demais, em sua maioria, não estudavam quando engravidaram.

Estes dados evidenciam que o abandono escolar, nestas comunidades, não está diretamente vinculado à gravidez precoce, principalmente no estado de Se. Nota-se também uma diferença entre as duas comunidades baianas, sendo que a área semirural se assemelha às comunidades sergipanas, sugerindo que as comunidades mais empobrecidas tendem à evasão escolar mais precocemente.

Com relação ao retorno aos estudos, raras foram as entrevistadas que mencionaram ter este objetivo em seus planos. A maioria alega não ter com quem deixar os filhos, apesar de 53\% das adolescentes de Se e $64 \%$ da Ba terem uma rede de apoio familiar para os cuidados com o filho (Tabela 4). Algumas afirmam não terem vontade de estudar, apesar de a maioria verbalizar que o estudo é muito importante para o futuro profissional, utilizando-se de frases clichês como "sem ele ninguém é nada”.

Quanto à situação empregatícia das gestantes/mães adolescentes, 90\% das entrevistadas de Se não estavam trabalhando quando foram entrevistadas e $62 \%$ delas nunca haviam trabalhado. $\mathrm{Na} \mathrm{Ba}$, o índice de desemprego quando da entrevista era superior ao de Se, pois 95\% das entrevistadas não exerciam nenhuma atividade profis-
Tabela 4 - Rede de suporte social das adolescentes da pesquisa

\begin{tabular}{ccc}
\hline Rede de suporte & $\begin{array}{c}\text { Bahia } \\
\mathbf{\%}\end{array}$ & $\begin{array}{c}\text { Sergipe } \\
\mathbf{\%}\end{array}$ \\
\hline Mãe da adolescente & 45 & 37 \\
Sogra da adolescente & 37 & 16 \\
Marido e sogra & 08 & 20 \\
Familiares & 28 & 27 \\
\hline
\end{tabular}

sional, tanto na área rural quanto na área urbana (Tabela S). Contudo, mais da metade das baianas já exercera alguma atividade profissional, em geral de caráter temporário como lavradoras,"”oficce girl”, vendedoras e empregadas domésticas. Tanto em Sergipe quanto naBahia a maioria das adolescentes que estavam trabalhando exerciam serviços domésticos.

Tabela 5 - Situação empregatícia das gestantes/ mães adolescentes

\begin{tabular}{ccc}
\hline Trabalha & $\begin{array}{c}\text { Bahia } \\
\text { \% }\end{array}$ & $\begin{array}{c}\text { Sergipe } \\
\mathbf{\%}\end{array}$ \\
\hline Não & 95 & 90 \\
Sim & 05 & 10 \\
\hline
\end{tabular}

É possível supor, a partir destes dados, que a escolarização não é a prioridade dessas jovens. $\mathrm{O}$ abandono escolar antecede à gestação precoce, e quando esta é citada como causa da interrupção dos estudos está mais diretamente relacionada ao significado social da gravidez na adolescência. A partir daí, muitas justificam a evasão escolar por questões morais, principalmente quando a gravidez ocorre antes do casamento.

Para Fávero e Mello², ao assumir o novo papel, o de mulher e mãe, as adolescentes abandonam a escola, desistem ou são desestimuladas a retornarem pois o seu novo papel é mais valorizado. A super-valorização do papel de mãe, a noção social da gravidez como realização da mulher ou como critério de saúde e maturidade, é ainda maior nas camadas sociais de baixa renda e, especialmente, naquelas onde há pouca expectativa profissional.

Outro fator que corrobora para este quadro é o fato de estas adolescentes serem filhas de mães que engravidaram na adolescência, exercendo geralmente atividades domésticas, apontando para a influência do contexto sóciofamiliar na vida dessas jovens, onde a gravidez precoce é percebida e esperada como algo natural, comum à comunidade.

\section{CONCLUSÃO}

Esse estudo indica que a gravidez/ maternidade na adolescência não deve ser sempre 
vista como a principal responsável pelas condições de subsistência a que está submetida grande parte das mulheres nas comunidades carentes. Em alguns casos, como nas comunidades aqui estudadas, a própria gravidez pode emergir como uma "solução” ante um contexto sócio-familiar que se impõe na forma de um "destino familiar" e ser, ela mesma, uma contingência a mais no universo de dificuldades que cronifica a situação em que se encontram os indivíduos das camadas populares, mantendo e reproduzindo a exclusão social ${ }^{4,5,7,10}$.

A perspectiva de que a gravidez precoce coloca as jovens em situação de risco sócio-econômico eminente é uma visão determinista e limitada: primeiro, ao não considerar a possibilidade de o sujeito reagir às contingências do ambiente de modo pessoal; segundo, ao não con- siderar a possibilidade de o ambiente estar construindo um valor social baseado na maternidade. Neste contexto, a falta de expectativas em relação ao estudo e ao trabalho pode distanciar as jovens de um investimento mais efetivo na escolarização, tendo como alternativa mais acessível a adultez e o exercício da maternidade.

No momento em que há uma dissonância entre a cultura da escola e a realidade objetiva dos sujeitos, a percepção dessa instituição como promotora de melhores condições de vida física se distancia das perspectivas das comunidades mais carentes. Abandonar a escola e não ter acesso a um trabalho melhor remunerado está na “ordem do dia”, independentemente da condição de ser adolescente grávida/mãe, embora essa condição possa antecipar o que aconteceria em algum momento da história dessas jovens.

\begin{abstract}
This research aimed lo investigate lhe education and employment situation of young girls who experience prematuro maternity and to establish a relation between teenager pregnancy and school dropout, comparing the educacional and employment reality of young mothers from communities in lhe states of Bahia and Sergipe. To achieve this, home visits were made to eighty pregnant teenagers or young mothers from poor communities in the two capitais. The results showed that more than half of them had already been out of school when they got pregnant and many of them left school after they discovered they were pregnant. Although they have been helped by the government, only few of them continued studying. Most of them did not work before the pregnancy; some of them started working after the maternity, mainly as housekeepers. It is possible to suppose that education is not linked with the culture of these communities. The social value of the woman is restricted to maternity, which is highly valued in the group and which gives them the adult status. Therefore, instead of viewing premature pregnancy as causing exclusion, our study showed that it seems to be caused by the social abandonment that victimizes these poor young girls.
\end{abstract}

Key-words: maternity. adolescence. formal education. Iabor.

\section{REFERÊNCIAS}

1 Muss RE. Teorias da adolescência. 5a ed. Belo Horizonte: Interlivros; 1969. cap. 5.

2 Fávero MH, Mello RM. Adolescência, maternidade e vida escolar. Psicol Teoria Pesq 1997; 13(1): 131-ó.

3 Gauderer EC. Crianças, adolescentes e nós: questionamentos e emoções. São Paulo: ALMED; 1987.

4 Esteves JR. Trajetórias de vida: repercussões da gravidez adolescente na biografia de mulheres que viveram tal experiência [dissertação]. Vitória (ES): Psicologia da Universidade Federal do Espirito Santo; 2003.

5 Catharino TR, Giffin K. Gravidez e adolescência: investigação de um problema moderno. In: Anais do $13^{\circ}$ Encontro Nacional de Estudos Populacionais; 2002; Ouro Preto/MG.
6 Bronfenbrenner U. A ecologia do desenvolvimento humano: experimentos naturais e planejados. Porto Alegre: Artes Médicas; 1996.

7 Fonsêca ALB. Práticas educativas no contexto familiar das mães adolescentes[dissertação]. Salvador(Ba): Faculdade de Educação da Universidade Federal da Bahia; 2000.

8 Novelino AM. Maternidade: um perfil idealizado. Cad Pesq. 1988; (65): 21-9.

9 Arcieri JBC. A mãe jovem e o seu primeiro bebê [tese]. São Paulo (SP): Instituto de Psicologia da Universidade de São Paulo; 1998.

10 Dadoorian D. A gravidez desejada em adolescentes de classes populares [dissertação]. Rio de Janeiro (RJ): Departamento de Psicologia da Pontifícia Universidade Católica; 1994.

Recebido em 20/01/2004

Modificado em 07/03/2004

Aprovado em 02/06/2004 
Uma boa comunicação entre os profissionais e os pais possibilita a obtenção de consenso sobre a divisão de responsabilidades quanto aos cuidados para com a criança. Isto evitará que os pais se sintam desconfortáveis ou pressionados a aceitar responsabilidades que eles não dominam e pode evitar que fiquem esquecidos e/ou subestimados pela equipe ${ }^{22}$.

Os profissionais geralmente requisitam dos pais que assumam os cuidados de higiene e alimentação de seu filho. Entretanto, eles nem sem- pre se preocupam em saber se os pais concordam, desejam ou se sentem à vontade existem inúmeras dificuldades decorrentes da inclusão da mãe/familiar não só como participante nos cuidados à criança, mas também como alguém que necessita de cuidados. Também há muitos locais em que há falta de condições organizacionais e de infra-estrutura. Pesquisas, principalmente experimentais, nessa área podem trazer novos elementos que favoreçam a interação, colaboração e respeito nos cuidados à criança e sua família.

\begin{abstract}
This work aims to contribute to the discussion concerning the assistance provided to the family of the hospitalized child through a theoretical reflection based on our experience in the arca. Our aim was to understand the family perspective in cases of child hospitalization, as well as the challenges posed to the nursing staff in learning how to approach the family in the hospitalization context. It was verified that the expectations and necessities of the family are different from those of the professionals involved who, in turn, encounter difficulties in giving support and guidance to the families. The absence of technical, scientific and operational conditions for providing appropriate assistance is one of the limiting factors. In order to implement an ideal model of assistance which takes into consideration the necessities of both the child and the family, it is necessary to invest in infrastructure and in the specific training of the professionals involved, thus changing the current reality of the assistance provided for hospitalized children.
\end{abstract}

Key words: family. hospitalized child. pediatric nursing.

\section{REFERÊNCIAS}

1. Thompson WR, Grusec JE. Estudo das primeiras experiências. In: Netto CdebSP, editor. O primeiro ano de vida e as experiências iniciais II. São Paulo: EPU; 1975. p.171-305.

2 Department. of Health and Social Security. Central Health Services Council. The welfare of children in hospital: report of the committee. London: Her Majesty s Stationery Office; 1959.

3 Valle ERM. A psicologia na formação e manutenção do desempenho da enfermeira pediatra. Rev Paul Enferm 1984;4(3): 105-8.

4 Bowlby J. Los cuidados maternos y la salud mental. Washington: Organización Panamericana de la Salud; 1968.

5 Brasil. Lei nº 8.069, de 13 de julho de 1990. Dispõe sobre o Estatuto da Criança e do Adolescente, e dá outras providências. 1990.

6 Collet N. Criança hospitalizada: participação das mães no cuidado [tese]. Ribeirão Preto: Universidade de São Paulo; 2001.

7 Runeson I, Hallstrom I, Elander G, Hermerén G. Children's needs during hospitalization: an observational study of hospitalized boys. Int J 15. Nurs Pract 2002; 8(3): 158-completar

8 Oliveira I, Angelo M. Vivenciando com o filho uma passagem difícil e reveladora: a experiência da mãe acompanhante. Rev Esc Enferm USP 2000; 34(2): 202-8.
9 Bezerra LFR, Fraga MNO. Acompanhar um filho hospitalizado: compreendendo a vivência da mãe. Rev Bras Enferm 1996; 49(4): 611-24.

10 Chiattone HBC. Relato de experiência de intervenção psicológica junto a crianças hospitalizadas. In: Camon VAA, editor. Psicologia hospitalar: a atuação do psicólogo no contexto hospitalar. São Paulo: Traço Ed.; 1984.

11 Bezerra SL, Veríssimo MLOR. A experiência de ser irmão de uma criança doente e hospitalizada: uma análise da literatura. Rev Soe Bras Enferm Pediatr 2002; 1(2): 29-35.

12 Miles MS, Burchinal P, Holditch-Davis D, Brunssen S, Wilson SM. Perceptions of stress, worry, and support in black and white mothers of hospitalized, medically fragile infants. J Pediatr Nurs 2002; 17(2): 82-8.

13 Noyes J. A critique of studies exploring the experiences and needs of parents of children admitted to paediatric intensive care units. J Adv Nurs 1998; 28(1): 134-41.

14 Scoffstall MC. Children's perceptions of hospital stressors. Nurs Times 1988; 84: 60.

15 Monteiro Filho L. O programa de hospitalização da criança acompanhada no hospital municipal Souza Aguiar: análise dos conflitos gerados com a equipe de saúde. J Pediatr 1998 ; 64(6): 242-7. 
16 Wernet M. Enfermagem e família - investindo no primeiro passo. Rev Bras Enf 2000; 53 (especial): 87-9.

17 Lima RAG, Rocha SMM, Scochi CGS, Callery P. Involvement and fragmentation: a study of parental care of hospitalized children in Brazil. Pediatr Nurs 2001 ; 27(6): 559-80.

18 Lacaz CPC, Tyrrell MAR. A enfermagem e o cuidar de crianças com câncer: uma jornada pelo simbólico a partir da realidade vivenciada pelo universo farniliar. Acta Paul Enferm 2003; 16(2): 33-40.

19 Shields L. The parent-staff interaction model of pediatric care. J Pediatr Nurs 2002; 17(ó):442-8.

20 Motta MGC. O ser doente no tríplice mundo da criança, família e hospital: uma descrição fenomenológica das mudanças existenciais. Florianópolis: Enfermagem PEN/UFSC; 1998.

21 Wernet M, Angelo M. Mobilizando-se para a família: dando um novo sentido à família e ao cuidar. Rev Esc Enferrn USP 2003; 37(1): 19-25.
22 Lissauer T, Clayden ( . Manual ilustrado de pediatria. $2^{\mathrm{a}}$ ed. Rio de Janeiro: Guanabara Koogan; 2003.

23 Darbyshire P. Living with a sick child in hospital: the experiences of parents and nurses. London: Chapman \& Hall; 1994.

24 Kristensson-Hallstrom I. Strategies for feelingsecure influence parents participation in care. J Clin Nurs 1999; 8(5): 586-92.

25 Behrman RE. Nelson - Tratado de pediatria. $16^{\text {a }}$ ed. Rio de Janeiro: Guanabara Koogan; 2002.

26 Shields L. A review of the literature from developed and developing countries relating to the effects of hospitalization on children parents. Int Nurs Rev 2001; 48(1): 29-37.

27 Schaffer P, Kenner IC. Comunication between nurse and hospitalized child. Soins Pediatr Pueric 2000; 194: 6-8.

28 Lima R. Enfermagem na assistência à criança com câncer. Goiânia: AB; 1995.

Recebido em 10/05/2004

Aprovado em 02/06/2004 